\title{
Oral health effect by dentifrice combined with extracts of Centella asiatica
}

\author{
Kyung Sook HWANG
}

Dept. of Dent. Tech., Shin Han Univ., Korea

Objectives: This experiment was contrived to find out the changed in oral condition index when standardized and regular use of the dentifrice is done according to the usage of dosage of the dentifrice.

Methods: After inspecting the result of acceleration of collagen forming and suppressing effect of inflammatory reaction by using the titrated extracts of Centella asiatica with In-vitro experiment, the remedying effect on oral cavity condition was to be obtained with dentifrice made with combined bamboo salt and those above through in-vivo experiment worked with 60 healthy bodied examinees for over 3 months.

Results: With the result of the experiment done at the lab, when the changes of improved index of oral cavity condition, MMP-1 formative inhibition analysis, and collagen analysis were obtained during the experiment periods, the titrated extract of Centella asiatica suppresses the damage of the tissue due to inflammatory response at the gingiva cell and after the inflammatory response, accelerated regeneration of the gingiva were clearly shown. Also, as with the result in clinical experiment, the effect was acceptable in reducing effect of dental plaque index and gingivitis index with dentifrice that only contains the titrated extract of Centella asiatica and also showed outstanding result in antibacterial activity.

Conclusion: The most effective improvement especially shown on the oral conditions were dentifrice that contains Dipotassium glycyrrhizinate, bamboo salt and titrated extracts of Centella asiatica.

Keywords: bamboo salt, centella asiatica, dipotassium glycyrrhizinate

Acknowledgement: This study is supported by Uniance's Central Research Institute.

Copyright (C) 2021. Korean Academy of Preventive Dentistry. All rights reserved.

This is an Open Access article distributed under the terms of the Creative Commons Attribution Non-Commercial License (http://creativecommons.org/licenses/ by-nc/4.0) which permits unrestricted non-commercial use, distribution, and reproduction in any medium, provided the original work is properly cited. 